India, ideas, casta, liberalismo, izquierda

\title{
CRUZAR EL RÍO CON LAS SANDALIAS PUESTAS: RADICALISMO ANTIESTATISTA Y CONSERVADURISMO SOCIAL EN LA INDIA BRITÁNICA
}

\author{
DANIEL KENT CARRASCO \\ King's College
}

Durante las últimas décadas del siglo XIX y las primeras del XX, una irresoluble contradicción entre la retórica radical dirigida a la emancipación nacional y la práctica política incapaz de cuestionar jerárquicas estructuras sociales de casta empapó el debate público desarrollado en el proyecto nacionalista anticolonial indio. Esto contribuyó a exacerbar una ambigüedad no resuelta entre, por un lado, un marcado radicalismo en lo que concierne a la oposición a las estructuras y las formas del Estado $\mathrm{y}$, por otro, el conservadurismo frente a las jerarquías internas de la sociedad durante este periodo.

En este artículo me interesa explorar la presencia de esta ambigüedad en el debate público de la India británica en las últimas décadas del siglo XIX y primeras del XX, a través del análisis y yuxtaposición de dos momentos de la historia intelectual de la India británica y, en especial, de Bengala. El primero, de sobra conocido, concierne a la crítica nacionalista a la pobreza generada por las políticas económicas del Estado colonial a lo largo del siglo XIX; el segundo, considerablemente menos estudiado, abarca un conjunto de escritos que abordan la relevancia del sistema de castas para entender la expansión en India de ideas y principios asociados con el radicalismo de izquierda de tintes socialistas a partir del tercer cuarto del siglo XIX.

Este artículo fue recibido por la dirección de la revista el 9 de septiembre de 2014 y aceptado para su publicación el 18 de mayo de 2015. 


\section{Críticas al liberalismo económico del Estado colonial}

A partir de la segunda mitad del siglo XIX, miembros de las élites nativas de la India británica moldearon una potente corriente de crítica al Imperio, basada en una inversión de los principales argumentos de la economía política europea, dirigida a mostrar el papel que la total falta de restricciones económicas y mercantiles impuestas por el Estado colonial jugaba en el empobrecimiento crónico de la población india. Los "portadores canónicos" de esta crítica fueron miembros de la intelligentsia occidentalizada, entre los que destacaban M. G. Ranade, R. C. Dutt y Dadabhai Naoroji. ${ }^{1}$

La más célebre formulación de este temprano nacionalismo económico se encuentra en la obra de Naoroji, quien, durante la década de 1870, elaboró una serie de postulados que llegarían a ser englobados con el nombre de la teoría del Drenaje de Riqueza, o Drain of Wealth Theory. Naoroji nació en 1835, en el seno de una familia parsi de Bombay, y pronto desarrolló una impresionante carrera política a lo largo de la cual jugó un papel central en la creación de la Asociación de las Indias Orientales y la de su organización sucesora, el Congreso Nacional Indio. Además de haber sido el primer profesor no europeo del prestigioso Elphinstone College, Dadabhai también fue el primer miembro "de color" del parlamento británico. En julio de 1870, en Bombay, Naoroji ofreció una conferencia bajo el título de "Las carencias y los medios de India" (The Wants and Means of India), en la que abiertamente ponía en tela de juicio los registros estadísticos del Estado colonial para argumentar que India estaba siendo gobernada de acuerdo con una política deliberadamente dirigida a su empobrecimiento. En ella, comparaba el drenaje de la riqueza de India al efectuado por Inglaterra en Italia en el siglo XIV, el cual había dejado a este país en un estado de "total desamparo", donde las "alimañas [pululaban] en abundancia, en las ropas y las camas. La comida común eran chícharos, raíces de helechos e incluso corteza de los árboles. [...] La población, escasa de por sí, se hallaba per-

${ }^{1}$ La expresión es de Manu Goswami, Producing India: From Colonial Economy to National Space, Chicago-Londres, University of Chicago Press, 2004, p. 210. 
petuamente disminuida por la pestilencia y la necesidad". ${ }^{2}$ Sirviéndose de un repertorio de poderosas metáforas que incluían la del "vampirismo económico", "la India sangrante" y la "economía caníbal”, Naoroji desarrolló una devastadora crítica, sustentada empíricamente, al colonialismo que pronto se popularizó en periódicos europeos y británicos. Su análisis fue citado por Marx en el volumen 3 de El Capital, y llegó a convertirse en una de las condenas al colonialismo europeo más célebres del siglo XIX. ${ }^{3}$

A partir de la popularización del trabajo de Naoroji, el problema de la pobreza de India se convirtió en uno de los temas centrales en el arsenal retórico de los nacionalistas anticoloniales indios. Naoroji inauguró un estilo de crítica que, en lugar de basarse en el cuestionamiento de la validez del liberalismo económico, se limitaba a señalar los efectos paradójicos que tenía en India. La pobreza de India aparecía como el resultado deliberado de la extracción de recursos fuera del país, en conjunto con una falta de interés por la inversión nacional. En un famoso pasaje, Naoroji afirmaba:

[Nuestra] condición es muy anómala, parecida a la de un niño al que un padre cariñoso regala un dulce, pero para el que, en su condición desahuciada, el mismo dulce actúa como un veneno y, [...] al irritar su débil estómago, lo hace vomitar más y le causa mayor agotamiento. En la presente situación de India, lo que para otras naciones resultan golosinas, aquí parece actuar como veneno. ${ }^{4}$

De acuerdo con el análisis de Naoroji, el modelo de economía política defendido por Mill se había torcido en India, lo

${ }^{2}$ Dadabhai Naoroji, Poverty of India, Londres, V. Brooks, Day and Son, 1878, p. 39. La traducción al español del fragmento del texto de Naoroji es mía, al igual que la del resto de los textos reproducidos en este artículo. "[v]ermin [were] in abundance in the clothing and beds. The common food was peas, vetches, fern-roots, and even the bark of trees. [...] The population, sparse as it was, was perpetually thinned by pestilence and want".

${ }^{3}$ Goswami, Producing India, op. cit., p. 227.

${ }^{4}$ Naoroji, Poverty of India, op. cit., p. 40. "[O] $\mathrm{Or}$ condition is a very anomalous one-like that of a child to which a fond parent gives a sweet, but to which, in its exhausted condition, the very sweet acts like a poison, and, as a foreign substance, by irritating the weak stomach makes it throw out more, and causes greater exhaustion. In India's present condition, the very sweets of every other nation appear to act on it as poison". 
que dio como resultado un régimen "no británico" ("Un-British rule"), incapaz de beneficiar a Gran Bretaña o a sus súbditos indios; sin embargo, y a pesar de que en los años siguientes nacionalistas de todo tipo hicieron eco de sus argumentos, el incremento de la preocupación por la pobreza de India no se tradujo en una mayor preocupación por los pobres de India. La pobreza de India, a ojos de los pensadores liberales contemporáneos de Naoroji, no aparecía como el signo de una injusticia generalizada, sino como la muestra de la humillación y la impotencia de las élites que integraban la cúpula del movimiento nacionalista. En sus escritos, Naoroji participa de una ambigüedad generalizada entre los miembros de las élites nativas de la segunda mitad del siglo XIX respecto del colonialismo que consistía en la coexistencia de un reconocimiento de los beneficios que había traído a India - relacionados con la educación, la libertad de expresión y el progreso tecnológico- y el lamento por la condición de inferioridad en la que los indios eran situados frente a los colonizadores. Aun así, la corriente crítica inaugurada por él contribuyó de manera fundamental a generar un espacio en el cual se gestó una animada conversación acerca de tres conjuntos de ideas que llegaron a ser centrales para el desarrollo del nacionalismo radical y de izquierda socialista en las primeras décadas del siglo Xx en India: la aversión al libre mercado, el enfático apoyo a la regulación estatal de la economía, y la defensa de la producción industrial local y las masas de India.

La denuncia de la pobreza artificialmente causada de India como plataforma para la defensa del proteccionismo, la organización de las masas, la nacionalización de las industrias y la planeación de la economía aparecen también de forma prominente en los escritos de Prithwis Chandra Ray publicados entre 1895 y 1905. En la primera sección de su libro The Poverty of India, Ray esboza una narrativa sobre la prosperidad comercial de India que, afirma, se remonta a los tiempos del rey Salomón, y que concluye con la política desequilibrada del comercio colonial durante el siglo XIX, inclinada a favor de la metrópoli. Ray declara que el grueso de las importaciones que ingresaron a India desde Europa durante aquel siglo estaba formado por "cosas de lujo que no responden a las necesidades primarias de los 
nativos del país”, tales como licores, cristalería y perfumes. A continuación reflexiona: "Si, tal y como nos han dicho, las formas de gobierno occidentales representativas, democráticas o de cualquier índole resultan inadecuadas para India, entonces podemos atrevernos a decir que los artículos de lujo británicos resultan cien veces más inadecuados para las necesidades de un pueblo simple y tropical". ${ }^{5}$

Al preguntar, de manera retórica, “¿Quién ha escuchado que una nación se vuelva próspera y rica intercambiando cosas necesarias para su vida por los lujos de otra?", 6 y afirmar que India sólo podría aspirar a "recuperar el brillo de la prosperidad y un lugar en el mundo civilizado" a través del proteccionismo y la planeación de la economía, Prithwis nos muestra un temprano ejemplo del impulso a favor de la recuperación de las industrias indígenas, característico del movimiento swadeshi. Por otra parte, amplía el rango de la crítica de Naoroji al sugerir que el drenaje de la riqueza no afectaba sólo a India sino al mundo entero. "El libre mercado - afirmaba - sólo es posible entre pueblos libres [...] Inglaterra es, hablando con franqueza, el único país libre en este mundo". ${ }^{8}$

El ejemplo más claro y doloroso de la pobreza generada por el Estado colonial tomaba forma en la recurrencia de las hambrunas que, durante la segunda mitad del siglo XIX, azotaron las regiones rurales de India. El historiador Romesh Chunder Dutt habla de un total de 15 millones de muertos a causa del hambre entre 1877 y $1900,{ }^{9}$ mientras que el inglés William Digby afirma que las muertes alcanzaron los 36 millones tan só-

${ }^{5}$ Prithwis Chandra Ray, The Poverty Problem in India: Being a Dissertation on the Causes and Remedies of Indian Poverty, Calcuta, Thacker, Spink, 1895, pp. 13-14. "If, as we are told, representative, democratic, or for the matter of that any western form of government is unsuited to India, we can make bold to say that British articles of luxury are hundred times more unsuited to the requirements of a simple and tropical people".

${ }^{6}$ Ibid., p. 17. "Whoever heard of a nation becoming prosperous and wealthy by exchanging its necessaries of life for the luxuries of another?". world".

${ }^{7}$ Ibid., p. 43. "[R]ecover a gleam of prosperity and hold a place in the civilised

${ }^{8}$ Ibid., p. 66. "Free trade is only possible between free peoples. [...] England, truly speaking is the only free country in this world".

${ }_{9}^{9}$ Romesh Chunder Dutt, The Economic History of India under Early British Rule, Londres, Kegan Paul, 1902, p. vi. 
lo entre 1890 y $1900 .{ }^{10}$ Para principios del siglo xx era común la descripción de India como una tierra de carestía y escasez. Lejos de cancelar la imagen orientalista de India como una tierra de encantamiento y sabiduría ancestral, el tropo de la hambruna la complementaba, y alimentaba las buenas intenciones de agentes europeos, que iban desde los militantes defensores del Imperio hasta los numerosos y bondadosos misioneros cristianos. En paralelo, sin embargo, este tema también sirvió para reunir a grupos nacionalistas de diferentes inclinaciones en la denuncia de la política económica del Estado colonial. Prithwis expresaba una opinión generalizada al afirmar que las hambrunas de India eran "hambrunas de dinero, nunca de alimentos". ${ }^{11}$

Uno de los momentos culminantes de la crítica nacionalista a la política económica del Estado colonial aparece en los escritos de R. C. Dutt, uno de los historiadores indios más leídos de principios del siglo Xx. Como miembro de la élite que integraba el prestigioso Servicio Civil Indio, Dutt recibió los beneficios de la educación occidental y las oportunidades laborales ofrecidas a los nativos que pasaban a formar parte de la maquinaria del régimen colonial. Tras una larga carrera de funcionario en India, Dutt se retiró en 1897 y migró a Londres, donde trabajó como profesor en su alma mater, el University College de la Universidad de Londres. Durante los siguientes seis años completó traducciones al inglés del Mahabharata y el $R a$ mayana, así como varios tomos acerca de la historia, la economía y la cultura de India. En el primer volumen de su historia de India bajo el dominio británico, publicado en 1904, Dutt retomó de manera vigorosa el argumento, para entonces ya clásico, de la responsabilidad del Estado colonial por la pobreza de India y la recurrencia de las hambrunas:

En India, el Estado interfiere con la acumulación de la riqueza a partir de la tierra, intercepta los ingresos y las ganancias de los trabajadores y crónicamente aumenta la renta sobre la tierra, lo que sume al cultivador en la pobreza permanente. En Inglaterra, Alemania, Estados Unidos, Francia y otros países, el Estado hace crecer el ingreso de las personas, amplía

${ }^{10}$ William Digby, "Prosperous" British India: A Revelation from Official Records, Londres, T. Fisher Unwin, 1901, p. 137.

${ }^{11}$ Prithwis Chandra Ray, Indian Famines: Their Causes and Remedies, Calcuta, Cherry Press, 1901, p. 32. "[A] famine of money, never of food". 
sus mercados, abre nuevas fuentes de riqueza, se identifica con la nación y se enriquece a la par de ella. En India, el Estado no ha promovido ninguna industria nueva ni revivido la antigua industria en pos del pueblo; al contrario, interviene continuamente para extraer el producto de la tierra. ${ }^{12}$

El trabajo de Dutt condensa los principales postulados de la crítica de Naoroji y Prithwis Chandra Ray. Además, en sus escritos encontramos un nuevo elemento que se agrega a la crítica nacionalista al Estado colonial y que contribuyó a la gestación del nacionalismo radical de izquierda en India; en su opinión, la incapacidad del Estado para proveer de bienestar a los indios nacía de su lejanía del "pueblo" de India y su excesivo afán por funcionar como el administrador de su riqueza antes que como el regente de la comunidad nacional. Durante las primeras décadas del siglo xx, los líderes e ideólogos más radicales del nacionalismo anticolonial —como Bal Gangadhar Tilak, Bepin Chandra Pal y Aurobindo Ghosh-dieron mucha importancia a la definición y la defensa del pueblo de India, al cual se hacía referencia a través del interés en las masas, los millones de pobres; en efecto, el movimiento swadeshi estaba basado en la organización y la potencia de estos millones, y en la promoción de prácticas masivas como la resistencia pacífica, la desobediencia civil y el boicot económico y político del Estado colonial.

A partir de 1920, con la expansión del marxismo, este impulso masivo fue incorporado por los esfuerzos dirigidos a la organización de los trabajadores y los sindicatos. Sin embargo, no fue sino hasta la década de 1930 cuando se llevaron a cabo esfuerzos por desarrollar una reflexión teórica consistente en torno de la adaptación del socialismo a India. Hasta entonces, las ideas "progresistas" que encontramos en la obra de auto-

${ }^{12}$ Romesh Chunder Dutt, India in the Victorian Age, Londres, Kegan Paul, 1904, p. xi. "In India the State virtually interferes with the accumulation of wealth from the soil, intercepts the incomes and gains of the tillers, and generally adds to its land revenue demand at each recurring settlement, leaving the cultivator permanently poor. In England, in Germany, in the United States, in France and other countries, the State widens the income of the people, extends their markets, opens out new sources of wealth, identifies itself with the nation, grows richer with the nation. In India the State has fostered no new industries and revived no old industries for the people; on the other hand, it intervenes at each recurring land settlement to take out of the produce of the soil". 
res como Naoroji, R. C. Dutt o Prithwis Ray relativas a la necesidad de poner al Estado al servicio del desarrollo socioeconómico de la población india habían permanecido eclipsadas por la predominancia de un sentimiento de innato derecho de liderazgo de las élites respecto de las masas. Los liberales indios de finales del siglo XIX daban por sentado su derecho de tutela sobre el resto de la población, lo cual interfería con la posibilidad de una reflexión sincera y sistemática sobre las jerarquías e inequidades en el seno de la sociedad india colonial. La crítica desarrollada por estos grupos estaba dirigida básicamente al funcionamiento y las políticas del Estado colonial, y dejaba fuera de su espectro a la sociedad india, la cual era vista como el dominio exclusivo de las élites nativas dominantes. En este sentido, es importante señalar que a pesar de la creciente sofisticación con la que se comenzaban a abordar temas relacionados con la política económica del Estado colonial y con el deterioro de India, los debates llevados a cabo en los círculos de la intelligentsia nacionalista entre 1880 y 1910 respecto a la pobreza del país fueron llevados a cabo mayoritariamente al margen de un cuestionamiento respecto a las diferencias de casta. No obstante, sí hubo intentos, si bien aislados, por repensar el sistema de castas a la luz de algunas de las ideas defendidas por los radicales europeos de inclinaciones izquierdistas durante estos años. Abordaremos algunos de estos intentos en la siguiente sección.

\section{Igualdad, pobreza y castas}

A lo largo de las últimas décadas del siglo XIX, algunos intelectuales indios comenzaron a interesarse por el contenido y las implicaciones del modelo de justicia social propuesto por radicales europeos de tendencias izquierdistas o socialistas. Durante esos años, sin embargo, el izquierdismo europeo era pensado en India principalmente en relación con el sistema de castas; o, mejor dicho, se recurría a las castas en un esfuerzo por pensar acerca de la creciente importancia de las ideas socialistas y de izquierda, y relacionarlas con la sociedad india. En las siguientes páginas exploraremos el origen de la ambigua relación de los 
pensadores nacionalistas indios que reflexionaron sobre estos temas.

Antes de 1910 es posible distinguir dos acercamientos divergentes entre las ideas y los preceptos relacionados con el izquierdismo y el socialismo, y las castas. Por un lado, el sistema de castas era alabado por algunos autores como una forma más realista y orgánica de organización social para India; por otro, y en un periodo anterior, figuras como Bankim Chandra Chatterjee abordaron la injusticia que permea los sistemas de los varna y las jati, y destacaron el potencial revolucionario de algunas ideas provenientes de Occidente, entre las cuales se mencionaba explícitamente el socialismo, o se aludía a discusiones afines de igualdad y justicia social.

$\mathrm{Al}$ año siguiente de la publicación del tratado de Naoroji, Poverty of India, el pensador bengalí Bankim Chandra Chatterjee publicó un ensayo titulado Samya, en el que se hacía una fuerte denuncia de las injusticias inherentes al orden social defendido por el brahmanismo relativas a cuestiones de casta y género. ${ }^{13}$ Bankim fue un personaje crucial en la conformación del pensamiento nacionalista que emergió durante la segunda mitad del siglo XIX a partir de la evolución y la adaptación de la agenda de reforma social defendida por organizaciones como el Brahmo Samaj. Chatterjee nació en el seno de una familia brahmín en 1838, y produjo una rica y variada obra escrita que sería de gran importancia para el desarrollo de diferentes corrientes nacionalistas, incluido el radicalismo de tintes religiosos de miembros del Congreso, como Tilak, y las reflexiones poético-políticas de Rabindranath Tagore. Al margen de la esfera de la política nacionalista, Bankim ha sido aclamado como "el verdadero creador de la novela bengalí, la prosa bengalí madura y seria, y el fundador de la crítica literaria y el periodismo [en Bengala]". ${ }^{14}$ Más que ninguna otra figura de su tiempo, Chatter-

${ }^{13}$ Para este trabajo consulté la traducción al inglés del original bengalí realizada por M. K. Haldar y titulada: "Bankim on Equality", la cual aparece editada en Bankim Chandra Chatterji y M. K. Haldar, Renaissance E Reaction in Nineteenth Century Bengal, Columbia, South Asia Books, 1977.

${ }^{14}$ Tanika Sarkar, Hindu Wife, Hindu Nation. Community, Religion, and Cultural Nationalism, Bloomington, Indiana University Press, 2001, p. 135. "[T] he real maker of the Bengali novel, of mature and serious Bengali prose, and the founder of literary journalism and criticism". 
jee estudió a fondo diversas corrientes del pensamiento occidental, entre las que destacan el utilitarismo británico defendido por Jeremy Bentham; asimismo, publicó numerosos estudios acerca de la literatura sánscrita clásica, y compiló comentarios de las escuelas de especulación metafísica hindú. Tanika Sarkar ha observado que su ensayo, Samya, puede ser leído como una temprana exploración y denuncia del modelo del homo bierarchicus de la sociedad brahmánica india; ${ }^{15}$ en efecto, el texto nos enfrenta a una fuerte crítica igualitaria del sistema de castas, que desaparecería de su obra posterior.

Bankim da inicio a su ensayo sobre la igualdad con la afirmación de que la diversidad es un hecho innegable de la naturaleza, cuya existencia no puede ni debe ser puesta en duda. En su opinión, los hombres y las mujeres nacen dotados de diferentes capacidades y habilidades, que es lo que genera su identidad; sin embargo, pronto aborda el problema que será el centro de su argumentación a lo largo del texto; a saber, la división creada artificialmente entre hombres "grandes" y "pequeños". Los cínicos, nos dice Bankim, proveen una explicación económica para esta división antinatural: el hombre pequeño lo es debido a su incapacidad para engañar, mientras que el hombre grande lo es sólo gracias a la falsedad, el robo, o la capacidad de obtener beneficio del robo o la muerte de un ancestro, $u$ otro tramposo. ${ }^{16}$ Conforme avanza el texto, Bankim elabora este planteamiento simplista y describe otro tipo de jerarquía y otra clase de "grandes hombres":

Gopal Thakur está agobiado por el hecho de que su hija no esté casada, y debido a su agobio, va rogando de casa en casa. Incluso a pesar de ser un gran hombre. ¿Por qué? Porque Gopal es un brahmín. Tú eres un shudra, no importa lo grande que puedas ser, tendrás que tocar el polvo de mis pies. [...] Gopal es pobre, iletrado, el peor ejemplo de humanidad, un pecador, y sin embargo es un gran hombre. ${ }^{17}$

${ }^{15}$ Ibid., p. 154.

${ }^{16}$ Chatterji y Haldar, Renaissance E Reaction in Nineteenth Century Bengal, op. cit., p. 150.

${ }^{17}$ Idem. "Gopal Thakur is burdened with his unmarried daughter-and because he is burdened with his unmarried daughter he is begging from door to door. Even so he is a big man. Why, because Gopal is a Brahmin. You are a Sudra-however big a man you may be, you will have to take the dust of my feet. [...] Gopal is poor, illiterate, a worse specimen of humanity, a sinner, yet he is a big man". 
En Samya, la falta de equidad es descrita en términos de una herida social que necesita la acción de cirujanos sociales para ser curada. Bankim menciona a los revolucionarios franceses Robespierre y Danton como dos ejemplos de cirujanos sociales, capaces de suplantar la diferencia artificial con la igualdad. ${ }^{18}$ No obstante, estos cirujanos por sí solos no son suficientes; necesitan ser guiados por alguien más, un avatar de la igualdad, de los cuales sólo tres han aparecido en la historia de la humanidad: el Buddha, Jesucristo y Rousseau. El primero apareció para sanar las inhumanas diferencias de casta institucionalizadas por el orden del dharma védico; el segundo, para eliminar la distinción entre maestro y esclavo sobre la cual se construyó el Imperio romano, y el tercero, para terminar con la separación entre clases altas y bajas reinante en la Francia absolutista. Bankim se preocupa por distinguir a Rousseau de los primeros dos, pues afirma que mientras los primeros "predicaban la verdad sagrada en la tierra", él no es un mensajero de la "verdad pura y sin adulterar", sino más bien un "mago de la palabra" y el padre de muchos hombres importantes - entre los que nombra a Proudhon, uno de los padres del primer socialismo-y generador de valiosas ideas como la del "comunismo", y las de "la tierra pertenece a todo" y lo "internacional". ${ }^{19}$

En un capítulo subsecuente, Bankim considera la situación del campesino bengalí, hundido en una posición de sujeción y endeudamiento crónico respecto del zamindar (terrateniente); sin embargo, se cuida de generalizar. La miseria del campesino indio, afirma, no es solamente la culpa del zamindar, al cual sería injusto catalogar como un perpetuo explotador. Bankim ofrece otras explicaciones para la ancestral miseria de los campesinos; una de ellas obedece a la ausencia de "ahorro social", un concepto similar a la idea marxista de la plusvalía, que representa, a los ojos de Bankim, el único camino para el progreso y la obtención del conocimiento. ${ }^{20} \mathrm{~A}$ través de un análisis histórico notablemente parecido al desarrollado por Marx, Bankim explica la emergencia, mediante un mecanismo de acumulación de capital, de dos clases: una dedicada al trabajo y otra, al cono-

\footnotetext{
${ }^{18}$ Ibid., p. 152.

${ }^{19}$ Ibid., pp. 159-163.

${ }^{20}$ Ibid., p. 177.
} 
cimiento. A continuación propone que tal distinción no debe ser necesariamente tomada como injuriosa para cualquiera de las clases, sino que puede, a la inversa, revelarse como benéfica, ya que presenta la posibilidad de organizar las diversas ocupaciones necesarias para el mantenimiento de toda sociedad. El conflicto, no obstante, se hace inevitable cuando el incremento de la población es superior al incremento de la riqueza, como en el caso de India a lo largo del siglo XIx: ${ }^{21}$ "Como resultado del avance de la civilización, los trabajadores indios comenzaron a llevar una vida miserable. La fertilidad de la tierra y el clima cálido [...] las dos causas de [este] avance [...] se tornaron las causas de las miserias del hombre común".22

A pesar de que la fase inicial del origen de la miseria del "hombre común" es caracterizada por Bankim como el resultado de una "ley natural", a ésta le sigue la instauración de una ley "antinatural", contenida en los shastra, y que sanciona la sujeción y opresión a los shudra por la clase intelectual, cuya riqueza se ha incrementado a lo largo de los siglos hasta colocarla en una posición de dominación justificada por el poder del ritual y el conocimiento. Este último paso cierra un círculo de desigualdad, formado por la "pobreza, la ignorancia y la esclavitud". ${ }^{23}$ Es la perpetuidad de este círculo precisamente lo que se encuentra en la raíz del atraso de India. Al hacer eco del proyecto del Brahmo Samaj y del pensamiento de figuras como Vidyasagar, ${ }^{24}$ Bankim vislumbra en la educación la única posible salida de este círculo vicioso, e insiste en que la jerarquía de castas se encuentra en el fondo de la miseria de India; sin embargo, en sus conclusiones, remarca el estatus natural de las diferencias y la imposibilidad de llegar a un verdadero estado de igualdad.

Tanika Sarkar afirma que en Samya, Bankim señaló la posibilidad de una agenda y una voz que prometían ser las más visionarias y más radicalmente humanitarias de entre las que ha-

${ }^{21}$ Ibid., p. 180.

${ }^{22} \mathrm{Ibid}$., p. 183. "As a result with the rise of civilization, the Indian workers began to live a miserable life. The fertility of the land and the warm climate-the two reasons for the rise of civilization became the causes of the miseries of the common man".

${ }^{23}$ Ibid., p. 182.

${ }^{24}$ Ibid., p. 202. 
bían surgido hasta entonces entre la intelligentsia bengali, ${ }^{25}$ no obstante, Bankim declaró más tarde sentirse arrepentido de haber escrito Samya, y expresó opiniones más afines al nativismo hindú que a pensadores como Rousseau; ${ }^{26}$ de hecho, el problema de las inequidades de casta y género desaparecieron prácticamente de su obra posterior. No obstante, Samya permanece como un documento importante y revelador para la historia de las ideas en India; en efecto, su tratamiento de las ideas políticas de Rousseau y su uso de ideas similares a las que se sitúan en el núcleo de la interpretación marxista son fascinantes; además, sus planteamientos preceden a los enfoques divergentes de Gandhi y Ambedkar. Por una parte, en Samya se observan rasgos de la posición defendida en años posteriores por Gandhi, según la cual la casta representa una categoría inherente al orden de la sociedad hindú. Al mismo tiempo, la mención del Buddha como uno de los tres avatares de la igualdad antecede a la insistencia del viejo Ambedkar en la conversión de la comunidad intocable al budismo, mientras que la insistencia de Bankim en la educación como el único medio de terminar con la injustica recuerda la enérgica propuesta de Ambedkar de "educar, educar, educar".

En 1899, veinte años después de la publicación de Samya, Swami Vivekananda (1864-1902) vincularía otra vez el problema de las castas a la reflexión en torno de la justicia social desarrollada por adeptos al socialismo y la izquierda en Europa. En marzo de 1899, en el marco de una reflexión acerca de los cambios sociales que se estaban gestando en India, Vivekananda lanzaba la siguiente pregunta:

¿Y dónde están aquellos a través de cuyo trabajo físico únicamente resultan posibles la influencia del brahmín, las proezas del kshatriya y la fortuna del vaishya? ¿Cuál es la historia de aquellos a quienes, siendo el verdadero cuerpo de la sociedad, se les designa en todo momento y en todos los países como de "nacimiento inferior"? ¿Para los que, en India, les ha sido prescrito el leve castigo: "Cortarle la lengua, arrancarle la piel" [...] los shudras, cuál es su destino en la vida? ${ }^{27}$

${ }^{25}$ Sarkar, Hindu Wife, Hindu Nation, op. cit., p. 156.

${ }^{26}$ Idem.

${ }^{27}$ Swami Vivekananda, The Complete Works of Swami Vivekananda, Calcuta, Advaita Ashrama, 1992, vol. 4, p. 466. "And where are they through whose physical labour only [sic.] are possible the influence of the Brahmin, the prowess of the Ksha- 
Durante los últimos años de su vida, Vivekananda popularizó la tradición del Vedanta y predicó una ambigua posición nacionalista que elaboraba y expandía el impulso de reforma de la sociedad hindú presente en los textos de Bankim y otros intelectuales bengalíes decimonónicos. En su comentario a las Leyes de Manu, Vivekananda expone su preocupación por la injusticia generada por el sistema de castas, y apunta a la inevitabilidad de su transformación por el bien de India. Dicha transformación, otra vez, ocurriría como resultado de la expansión de la educación y los cambios propiciados por la dinámica sociopolítica de Occidente.

Los shudras de otros países, al parecer, han comenzado a despertar; pero carecen de la educación adecuada y conocen sólo el odio para los de su propia clase $[. .$.$] ¿De qué les sirve superar en número a las otras cla-$ ses por grandes márgenes? La unidad, mediante la cual diez hombres ostentan la fuerza de un millón, está aún lejos de los shudras; por ende, de acuerdo con la ley de la naturaleza, los shudras constituyen invariablemente la raza sometida.

Pero hay esperanza. Con el poderoso paso del tiempo, los brahmín y las otras castas altas están siendo arrojadas al bajo estatus de los shudras, y ellos son elevados a rangos superiores. ${ }^{28}$

En sus escritos, Vivekananda no usa el término shudra en referencia al orden jerárquico y organicista promulgado por el sistema del varnashramadharma, sino para referirse a las masas empobrecidas y explotadas, no sólo de India sino del mundo entero. En su opinión, la posibilidad de una transformación social que acabara con la pobreza era posible y se hacía patente en las maneras en las que los shudras de todo el mundo parecían

triya, and the fortune of the Vaishya? What is their history, who, being the real body of society, are designated at all times in all countries as 'base-born'? For whom kind in India prescribed the mild punishment 'Cut out his tongue, chop off his flesh' [...] the Shudras, what is their lot in life?".

${ }^{28} \mathrm{Ibid}$., pp. 466-467. "The Shudras of countries other than India have become, it seems, a little awake; but they are wanting in proper education and have only the mutual hatred of men of their own class-a trait common to Shudras. What avails it if they greatly outnumber the other classes? That unity, by which ten men collect the strength of a million, is yet far away from the Shudra; hence, according to the law of nature, the Shudras invariably form the subject race. But there is hope. In the mighty course of time, the Brahmin and the other higher castes, too, are being brought down to the lower status of the Shudras, and the Shudras are being raised to higher ranks”. 
estar revolucionando el orden social establecido en su propio beneficio, y buscaban ser "elevados a rangos superiores"; sin embargo, crucialmente, el empoderamiento de los shudras no significaba un cambio en su naturaleza esencial o de su lugar en la sociedad; antes bien, representaba una revolución de clase en la cual es posible encontrar indicios del proyecto de la dictadura del proletariado. De la vinculación entre la injusticia de las castas y la de la pobreza, Vivekananda extrae la predicción de una revolución social venidera que liberaría a los shudras a través de la potencia de las ideas del socialismo, el anarquismo e, incluso, el nihilismo:

No obstante, llegará el momento de la sublevación de la clase de los shudras, con todo y su naturaleza de shudras; es decir [...] que llegará un momento en que los shudras de todos los países, con su naturaleza y hábitos innatos de shudras - sin convertirse en vaishyas o khsatriyas, sino como shudras - conquistarán la supremacía absoluta en todas las sociedades. [...el] socialismo, el narquismo, [el] nihilismo y otras sectas similares son la vanguardia de la revolución social que vendrá. ${ }^{29}$

El periodo de mayor actividad de Vivekananda tuvo lugar en la década de 1890, los años previos al levantamiento swadeshi, cuyo origen se situó en Bengala. Durante este tiempo, Vivekananda permaneció ajeno a la actividad del Congreso y otros grupos políticos nacionalistas, y se concentró principalmente en la promoción de las virtudes de la espiritualidad, la metafísica y los principios de la cultura sánscrita. No obstante, en sus reflexiones respecto de la injusticia cometida en contra de las castas bajas, y en su extrapolación de esta injusticia de varna a otros países en el mundo, es posible descubrir las formas en las que, durante las últimas décadas del siglo XIX, la crítica a la pobreza creada por la política económica liberal del Imperio en India comenzaba a extenderse más allá del ámbito de la economía - como sucedía en la teoría del drenaje de la riqueza- y a ser pensada en términos de la injusticia social que generaba.

${ }^{29} \mathrm{Ibid} .$, 477. "Yet a time will come when there will be the rising of the Shudra class, with their Shudra-hood; that is to say [...] a time will come when the Shudras of every country with their inborn Shudra nature and habits-not becoming in essence Viashya or Kshatriya but remaining as Shudras-will gain absolute supremacy in every society. [...] Socialism, Anarchism, Nihilism, and other like sects are the vanguard of the social revolution that is to follow". 
En contraste con los argumentos de Vivekananda que relacionaban la injusticia del sistema de castas con el espectro de la revolución propagada por el socialismo, en los escritos del menos conocido Kailas Chandra Kanjilal encontramos un acercamiento más pragmático al problema de las castas. En su libro An Essay on the Poverty of India: Its Causes and Remedies, publicado en 1893, Kanjilal argumenta que la casta "en términos estrictos [...] no representa otra cosa que la clasificación de los trabajadores y los obreros". La "clasificación" de las castas estaba,

[...] sin duda, calculada para mejorar las habilidades de los obreros, ya que los obliga a limitarse, generación tras generación, a su rama respectiva de la industria, a pesar de que esto a menudo les causa sufrimiento cuando deja de haber demanda por su trabajo. El uso de la maquinaria y la importación de artículos extranjeros han dejado a la mayoría en el desempleo [...] El resultado es que muchos [..., ] ya que deben sobrevivir, se insertan en la sociedad, necesariamente cometiendo delitos de distinto tipo. ${ }^{30}$

El problema de la pobreza, sin embargo, no sólo afectaba a las castas o las clases bajas, sino que además permeaba toda la sociedad como resultado de las inequidades generadas por el Estado colonial. Kanjilal tenía una opinión positiva del Imperio, marcada por su afinidad con la concepción hobbesiana del Estado. En su opinión, había que agradecer al Estado colonial por haber terminado con formas de anarquía en las que el miedo al vecino impedía la correcta inversión del capital, lo cual se transformaba en un obstáculo para la generación de riqueza. Según él, "la magia de la propiedad [privada, era capaz de convertir] la arena en oro y la mera piedra en un jardín". ${ }^{31}$ Kanjilal

${ }^{30}$ Kailas Chandra Kanjilal, An Essay on the Poverty of India: Its Causes and Remedies, Hugli, Hari Dass Paul, 1893, pp. 3-4. "[S]trictly speaking [...] is no other than a classification of workmen and labourers. This classification is, no doubt, calculated to improve the workmen's skill, as it makes them confine themselves from generation to generation to their respective branches of industry but it often causes them much suffering when their works are no longer in demand or paying. The use of machinery and the importation of foreign articles have thrown most of them out of employment. [...] The result is that a lot of idle hands as they must live, is necessarily let loose upon society to do it mischief in various ways [sic.]".

${ }^{31} \mathrm{Ibid}$., p. 13. "[T] $\mathrm{T}$ e magic of property (was capable of turning) sand into gold and a bleak stone into a garden". 
compartía la opinión de autores como Prithwis Chandra Ray y Romesh Dutt, quienes creían que el Estado debía ocuparse del manejo de la economía, la promoción de la industria local y la protección de los trabajadores y los pobres de India. Por otro lado, su opinión de las castas y la erradicación de la pobreza era similar a la de Vivekananda, pero se estructuraba en mayor sintonía con los debates económicos de la época que con los preceptos de la cultura sánscrita. La casta, creía, "debía reorganizarse de acuerdo con principios [...] liberales”. Lo que significaba, a grandes rasgos, que las castas no debían estar definidas por nacimiento, sino por el mérito:

Nosotros no apoyamos la anulación de las distinciones de casta y la mezcla imprudente en la sociedad. En las sociedades de todas las nacionalidades, tal y como se encuentran constituidas actualmente, debe haber aryas y sudras [...] patricios y plebeyos, siempre que la educación [...] no se filtre hacia los estratos más bajos de la sociedad. La educación y el valor moral, y no el mero accidente del nacimiento, deberían ser el estándar de la distinción de castas. Ni el principio de la hermandad universal que profesa simpatía respecto a objetos que no la merecen, ni la extrema tendencia [...] que produce hábitos de exclusividad y enajenación y el odio de todo lo nativo, son, a nuestro juicio, recomendables. Las pretensiones de las clases sacerdotales o brahmánicas, que esperan que todos se postren ante ellos y les ofrezcan obediencia servil, son altamente absurdas. ${ }^{32}$

Las inclinaciones de Kanjilal, claramente, no eran revolucionarias y, a diferencia de Vivekananda, nunca predijo un alzamiento causado por la pobreza en India. Al mismo tiempo, y anticipándose a un argumento muy popular entre aquellos detractores de la política estatal de reservaciones basada en las castas y aplicada luego de las recomendaciones de la Comi-

${ }^{32} \mathrm{Ibid}$., pp. 7-8. "We are not for doing away with caste distinctions and mixing society pell-mell. In the society of every nationality, constituted as it is at present, there must be Aryas and Sudras, [...] Patricians and Plebeians so long as education, which is the common leveller, commencing from the higher orders, is not filtered down to the lowest stratum of society [sic.]. Education and moral worth, and not the mere accident of birth, should be the standard of caste-distinction. Neither the principle of universal brotherhood, professing sympathy towards objects not deserving of it, nor the extreme denationalising tendency producing habits of exclusiveness and estrangement, and hating everything native, recommends itself to our judgement. The pretensions of the Sacerdotal or Brahmanical class, expecting all other classes to postrate [sic.] themselves before and offer it servile obeisance, are highly absurd". 
sión Mandal por el gobierno de V. P. Singh, en 1989, Kanjilal insistía en la necesidad de basar la distinción en el mérito de las personas y no en la pertenencia a una comunidad otorgada por nacimiento.

La tendencia crítica frente al esquema de las castas de la que participaron, aunque sólo fuera tímidamente, Bankim Chandra Chatterjee y Vivekananda tuvo su reverso en los escritos de autores que defendían el orden de las varnas como una alternativa más realista y legítima que aquellos modelos de justicia social contenidos en las doctrinas occidentales, como el socialismo. Un ejemplo notable de esta tendencia aparece en el panfleto de Satish Chandra Gan, The Caste System of India. A Practical Form of Socialism, publicado en Dibrugarh, en 1907. En este texto, Gan reemplaza la utopía socialista de la igualdad social con lo que denomina el acercamiento pragmático del hindú a la naturaleza humana, "tal y como es y no como debería ser". ${ }^{33}$ En un esfuerzo por legitimar las jerarquías del sistema de castas y presentarlo como una alternativa a lo que concebía como la nebulosa propuesta socialista, Gan plantea lo siguiente:

[El] socialismo y el sistema de castas tratan de proveer para todos; aquél insiste en el principio de la justicia y éste, en el de la necesidad; aquél nace de un sentimiento de obligación, mientras que éste, de un interés por la existencia propia. La idea socialista es ciertamente más elevada, pero la de la casta es en realidad más resistente. Aquélla es el resultado de la idea de la hermandad del hombre, mientras que ésta resulta de la unión orgánica de todas las cosas. El más grande logro del último siglo ha sido la abolición de la esclavitud. Lo cual sólo fue posible cuando se probó satisfactoriamente que respondía al interés del esclavista, y no meramente a su deber, acatarla. ${ }^{34}$

${ }^{33}$ Satish Chandra Gan, The Caste System of India. A Practical Form of Socialism, Dibrugarh, s. e., 1907, p. 14.

${ }^{34}$ Ibid., p. 13. Itálicas en el original. "[S]ocialism and the caste system try to make provision for everyone, the former insisting this as a matter of justice and the latter as a necessity; the former from the sentiment of duty and the latter from the interest of self-existence. The socialistic idea is certainly bigher but that of caste is certainly stronger. The former is the outcome of the idea of brotherhood of man, and the latter of that of organic union of all created things. The greatest achievement of the last century was the abolition of slavery. This was only found possible when it was proved to satisfaction that it was the interest of the slave owners and not simply his duty to do so". 
Según Gan, el socialismo, "tal y como fue expuesto en un inicio, era simplemente esto: toda la nación debía comportarse como una gran familia extensa hindú. Todo el ingreso individual debía ser depositado en un tesoro común y distribuido de manera equitativa entre los miembros" ${ }^{35}$ Esta armoniosa y jerárquica sociedad, resultado de la "unión orgánica de todas las cosas", es descrita como el fruto de la evolución gradual de la civilización hindú a lo largo de miles de años, y alabada como una forma de organización inigualable.

El socialista, aunque bien intencionado, parece desear, debido a su poca experiencia, que este poderoso río de egoísmo, con su lodo, mugre, corrientes, remolinos y arenas movedizas [...] algún día fluya ante él de tal manera que sea capaz de cruzarlo sin tener que quitarse las sandalias. El antiguo hindú, por otro lado, conocía bien lo fútil de tal expectativa, y estaba empeñado en el correcto desarrollo de la cabeza, los brazos y el cuerpo que le permitiría cruzar. ${ }^{36}$

El irónico y pragmático autor de este texto culpa al socialismo y su doctrina de la lucha de clases de la destrucción del tejido social europeo. A pesar de haber sido escrito más de diez años antes de la popularización de la insistencia estratégica de Gandhi en la política de la no violencia, Gan basaba su rechazo al socialismo precisamente en el mismo cimiento usado por Gandhi para desprestigiar la praxis y la ideología de socialistas y comunistas indios durante la década de 1930. Ambos, Gan y Gandhi, veían en el antagonismo de clases una amenaza a la integridad de la sociedad hindú y, por extensión, de la civilización india. Al referirse a la organización socioeconómica de la sociedad en términos de la administración del "tesoro común" de una familia extendida hindú, Gan parece preceder a los ar-

${ }^{35}$ Ibid., p. 5. "[A]s it was expounded at first, was simply this: the whole nation was to form as if one big Hindoo joint family. The whole individual earning were to be deposited in a common treasury to be distributed equally between all the members".

${ }^{36}$ Ibid., pp. 20-21. "The socialist, though well intentioned with his short experience appear to hope that this powerful river of selfishness with its mud, dirt, eddies, whirl pools, quicksands (sic.) [...] will one day flow past when he will be able to cross it with his slippers on. The ancient Hindoo, on the other hand, was well aware of the futility of such an expectation, and was bent on the proper development of the head arms body and feet (sic) to be able to swim across it". 
gumentos de Gandhi acerca de la justicia económica. En ambos casos se supone la existencia implícita de un fiduciario ${ }^{37}$ capaz de promulgar la justicia en la distribución de los recursos. Gandhi lo describiría en los siguientes términos: "[El] hombre rico será respetado en la posesión de su riqueza, la cual usará de manera razonable de acuerdo con sus necesidades personales, y sobre la cual actuará como el fiduciario para que el resto sea utilizado por la sociedad. Este argumento da por supuesta la honestidad del fiduciario". ${ }^{38}$

En la primera sección se argumentó que el origen de las ideas sobre el papel del Estado en la regulación de la economía, la restricción del libre mercado y la promoción de la industria india hallados en la obra de Naoroji y sus contemporáneos, y que más tarde se convertirían en planteamientos centrales del radicalismo nacionalista indio de principios del siglo xx, debe ser pensado como el resultado de la amplia crítica al liberalismo económico del Estado colonial desarrollada a partir de la década de 1870. La segunda sección se enfocó en la discusión de un conjunto de ideas concernientes a la relación entre la pobre$\mathrm{za}$, la casta y la igualdad llevada a cabo en las últimas décadas del siglo XIX y primeras del xx. La intención principal en la segunda sección ha sido mostrar que, durante esas décadas, existieron reacciones encontradas acerca de la relevancia de las ideas radicales del proyecto, encabezado por las élites, de reforma de la sociedad india, muchas de las cuales se enfocaron en la casta como la categoría central para el análisis del problema de la justicia social. Por un lado, conocidos personajes como Vivekananda y Bankim denunciaron las injusticias del sistema de castas, mientras que, por otro, autores de menor fama desarrollaron argumentos que precedían a la postura gandhiana, y defendían el sistema de castas como una alternativa de organización social más pragmática y eficiente que, además, aseguraba la supervivencia de la sociedad hindú. En este sentido, Satish Chandra Gan declaraba que aquellos hombres que denuncia-

${ }^{37} \mathrm{El}$ término inglés usado por Gandhi es trustee.

${ }^{38}$ M. K. Gandhi, "Equal Distribution”, Harijan, 25 de agosto de 1940. “(R)ich man will be left in possession of his wealth, of which he will use what he reasonably requires for his personal needs and will act as a trustee for the remainder to be used for society. In this argument honesty on the part of the trustee is assumed”. 
ban el sistema de castas como maligno no hacían más que "recomendar la decapitación como la cura más segura para el dolor de cabeza". ${ }^{39}$

\section{Conclusiones: en contra del Estado, a favor de la sociedad}

A partir de la primera década del siglo xx, el impulso nacionalista anticolonial en la India británica entró en una fase combativa identificada con el ideal del swadeshi y la exigencia inflexible del swaraj, o autogobierno. De las agitaciones y las campañas llevadas a cabo durante los primeros años del siglo emergieron los principales preceptos e ideales que fueron enarbolados por el programa de los sectores más radicales del Congreso Nacional Indio a partir de la década de 1920. En este programa fue de gran importancia la exigencia de la independencia inmediata, así como el uso de una retórica dirigida a la movilización popular de las masas y caracterizada por la defensa abstracta de ideales de emancipación y revolución. En años posteriores, a partir de la creciente aceptación del marxismo entre ciertos sectores del movimiento nacionalista, los argumentos centrales de la crítica decimonónica al liberalismo económico del imperio -relacionados con la denuncia del libre mercado y con el interés en responsabilizar al Estado por la organización del desarrollo económico y la defensa de la industria local - fueron retomados por una nueva generación de nacionalistas que se describieron a sí mismos como izquierdistas, entre los que destacan Jawaharlal Nehru, Subhas Chandra Bose y Jayaprakash Narayan. Los integrantes y los defensores de este impulso nacionalista vincularon la exigencia del autogobierno, o swaraj, con la idea de la autonomía de la sociedad india y su emancipación respecto al inflexible y explotador Estado colonial. Como resultado del antiestatismo ferviente de este nacionalismo anticolonial, durante las décadas de 1930 y 1940, estos sectores jóvenes del movimiento nacionalista desarrollaron un proyecto de transformación social basado en la superación del Estado colonial y la defensa

\footnotetext{
${ }^{39}$ Gan, The Caste System of India, op. cit., p. 5. "[R]ecommend decapitation as the surest cure for headache".
} 
de un Estado nacional independiente, como el garante de un desarrollo socioeconómico incluyente.

Sin embargo, si bien esta nueva generación de nacionalistas indios adoptó y defendió los principales postulados de la crítica de las élites nacionalistas de finales del siglo XIX, representadas por figuras como Dadabhai Naoroji o R. C. Dutt, también extendieron la incapacidad de estas élites decimonónicas de cuestionar efectivamente las inequidades dentro de la sociedad india. En este sentido, además de aceptar los postulados de la teoría del drenaje de la riqueza - desarrollada a partir de una robusta crítica al funcionamiento del Estado colonial que exigía su transformación-, el nacionalismo radical indio de principios del siglo Xx también adoptó una serie de posturas conservadoras relacionadas con la posibilidad de la transformación de la sociedad, cuya organización era concebida principalmente frente a la jerarquía de castas, defendida durante el siglo anterior. Así, es posible afirmar que el radicalismo del nacionalismo anticolonial indio a partir del movimiento swadeshi-que defendió diversos programas que iban desde el socialismo revolucionario hasta la sarvodaya gandhiana- representó un programa revolucionario sólo en términos antiimperialistas y respecto de la transformación de las estructuras del Estado, mientras que permaneció profundamente conservador en su postura ante las jerarquías internas de la sociedad india.

Dirección institucional del autor:

King's India Institute

King's College, London

Strand, London

WC2R 2LS, United Kingdom

danielkentca@gmail.com

\section{Bibliografía}

CHATTERJi, Bankim Chandra y M. K. Haldar, Renaissance $\mathcal{E}$ Reaction in Nineteenth Century Bengal, Columbia, South Asia Books, 1977.

Digby, William, "Prosperous" British India: A Revelation from Official Records, Londres, T. Fisher Unwin, 1901. 
Dutт, Romesh Chunder, India in the Victorian Age, Londres, Kegan Paul, 1904.

DutT, Romesh Chunder, The Economic History of India under Early British Rule, Londres, Kegan Paul, 1902.

GAN, Satish Chandra, The Caste System of India. A Practical Form of Socialism, Dibrugarh, s. e., 1907.

GANDHI, M. K., "Equal Distribution”, Harijan, 25 de agosto de 1940.

Goswami, Manu, Producing India: From Colonial Economy to National Space, Chicago-Londres, University of Chicago Press, 2004.

KanjILAL, Kailas Chandra, An Essay on the Poverty of India: Its Causes and Remedies, Hugli, Hari Dass Paul, 1893.

Naoroji, Dadabhai, Poverty of India, Londres, V. Brooks, Day and Son, 1878.

Ray, Prithwis Chandra, Indian Famines: Their Causes and Remedies, Calcuta, Cherry Press, 1901.

Ray, Prithwis Chandra, The Poverty Problem in India: Being a Dissertation on the Causes and Remedies of Indian Poverty, Calcuta, Thacker, Spink, 1895.

SARKar, Tanika, Hindu Wife, Hindu Nation. Community, Religion, and Cultural Nationalism, Bloomington, Indiana University Press, 2001.

ViveKanANDA, Swami, The Complete Works of Swami Vivekananda, Calcuta, Advaita Ashrama, 1992, vol. 4. 
